\title{
Patterns of Perceived Partner Responsiveness and Well-Being in Japan and the United States
}

\author{
Duygu Tasfiliz and Emre Selcuk \\ Middle East Technical University
}

\author{
Richard B. Slatcher and Elena F. Corriero \\ Wayne State University
}

\author{
Gul Gunaydin \\ Bilkent University
}

\author{
Anthony D. Ong \\ Cornell University
}

\begin{abstract}
Quality of marital relationships is consistently linked to personal well-being. However, almost all of the studies linking marital processes to well-being have been conducted in Western (particularly North American) countries. Growing evidence shows that perceived partner responsiveness is a central relationship process predicting well-being in Western contexts but little is known about whether this association generalizes to other countries. The present work investigated whether the predictive role of perceived partner responsiveness in well-being differs across the United States and Japan-2 contexts with contrasting views on how the self is conceptualized in relation to the social group. A large life span sample of married or long-term cohabiting adults $(n=3,079$, age range $=33-83$ in the United States and $n=861$, age range $=30-79$ in Japan) completed measures of perceived partner responsiveness, hedonic and eudaimonic well-being, and demographic (age, gender, education) and personality (extraversion and neuroticism) covariates known to predict well-being. Perceived partner responsiveness positively predicted hedonic and eudaimonic well-being both in the U.S. and in Japan. However, perceived partner responsiveness more strongly predicted both types of well-being in the United States as compared with Japan. The difference in slopes across the 2 countries was greater for eudaimonic as compared with hedonic well-being. The interaction between perceived partner responsiveness and country held even after controlling for demographic factors and personality traits. By showing that the role of perceived partner responsiveness in well-being may be influenced by cultural context, our findings contribute to achieving a more nuanced picture of the role of relationships in personal well-being.
\end{abstract}

Keywords: perceived partner responsiveness, culture, hedonic well-being, eudaimonic well-being, marriage

What makes people happy across the globe? Social relationships are listed as a major source of happiness by respondents across different parts of the world from North America and Western Europe to Africa and East Asia (Crossley \& Langdridge, 2005; Furnham \& Cheng, 2000; Lu \& Gilmour, 2004; Pflug, 2009). Growing empirical evidence supports these

Duygu Tasfiliz and Emre Selcuk, Department of Psychology, Middle East Technical University; Gul Gunaydin, Department of Psychology, Bilkent University; Richard B. Slatcher, Department of Psychology, Wayne State University; Elena F. Corriero, Department of Communication, Wayne State University; Anthony D. Ong, Department of Human Development and Weill Medical College, Cornell University.

The MIUDS II study was supported by National Institute on Aging Grant P01-AG020166, and the MIDJA study was supported by National Institute on Aging Grant 5R37AG027343-02.

A version of this article was presented at the 23rd International Congress of the International Association of Cross-Cultural Psychology in July 2016, Nagoya, Japan.

Correspondence concerning this article should be addressed to Emre Selcuk, Department of Psychology, Middle East Technical University, B45 Human Sciences Building, Ankara, Turkey. E-mail: semre@metu.edu.tr lay beliefs, with studies showing that the quality of social bonds exerts both immediate and lasting influences on well-being (Feeney \& Collins, 2015). One particularly potent relational process that contributes to adult health and well-being is perceived partner responsiveness - that is, the extent to which individuals believe that their romantic partner or spouse understands, appreciates, and cares for them (Reis, 2012; Selcuk, Karagobek, \& Gunaydin, in press; Slatcher \& Selcuk, 2017). Much of the existing research on the role of perceived partner responsiveness in personal well-being, however, has been conducted with North American samples. How strongly does perceived partner responsiveness predict well-being in nonWestern samples? The aim of the present study was to address this question by investigating whether the predictive role of perceived partner responsiveness in well-being differs across the United States and Japan-two contexts with contrasting views on how individuals relate to and are affected by their social world (Markus \& Kitayama, 2010).

\section{Perceived Partner Responsiveness and Well-Being}

Perceived partner responsiveness has been identified as a central process affecting the extent to which romantic relationships are 
satisfying and intimate (Reis, 2013). When individuals perceive their partner as caring, understanding, and appreciating, they are more likely to self-disclose and to react responsively to their partner's disclosures. When this process is enacted reciprocally and mutually, it reinforces the development and maintenance of intimacy and satisfaction in the relationship (Laurenceau, Barrett, \& Pietromonaco, 1998; Reis \& Shaver, 1988). Indeed, studies have shown that perceived partner responsiveness reliably predicts relationship intimacy with small-to-medium effect sizes (Debrot, Cook, Perrez, \& Horn, 2012; Otto, Laurenceau, Siegel, \& Belcher, 2015), and relationship satisfaction with medium-to-large effect sizes (Fekete, Stephens, Mickelson, \& Druley, 2007; Gable, Reis, Impett, \& Asher, 2004).

One consequence of maintaining a relationship with a responsive partner is improved personal well-being. Much of the extant research investigating the influence of responsiveness on wellbeing has been motivated by attachment theory (Bowlby, 1988). According to attachment theory, responsiveness of parents in infancy and childhood culminates in a sustained sense of felt security, which in turn promotes happiness and well-being in the long-term. In adulthood, spouses or long-term romantic partners are thought to assume the role of primary attachment figures and their responsive support is considered a key determinant of personal well-being.

When individuals encounter stressful experiences, responsive support from spouses serves to alleviate distress and restore feelings of security (Cutrona \& Russell, 2017). Indeed, contact with responsive partners has been argued to be the most effective way of alleviating negative affect during stressful experiences (Beckes \& Coan, 2015). Studies with North American and Western European samples have shown that perceiving partners as responsive predicts greater sustainability of positive affect or alleviation of negative affect when disclosing a stressful experience (Collins \& Feeney, 2000; Maisel \& Gable, 2009) and working on a challenging task (Feeney, 2004). The stress-buffering role of perceived partner responsiveness not only contributes to decreases in negative affect and increases in positive affect, but also allows individuals to devote their resources to autonomously and purposefully engaging with their daily environment, learning new things, and growing as a person, even in the face of adversity (Mikulincer \& Shaver, 2007).

The role of perceived partner responsiveness in well-being is not only limited to alleviating stress reactivity but extends to augmenting well-being in positive contexts (Cutrona \& Russell, 2017). For example, perceived partner responsiveness to positive selfdisclosures (e.g., sharing a success) increases and sustains positive affect and life satisfaction (Gable, Gosnell, Maisel, \& Strachman, 2012; Otto et al., 2015), even after controlling for the initial positivity of the shared information (e.g., Gable \& Reis, 2010). Moreover, perceived partner responsiveness when discussing personally important goals predicts increases in positive affect and perceived likelihood of goal attainment (Feeney, 2004). Increased confidence in goal attainment and the sense of security that comes with the belief that the partner will be available and responsive if things go wrong elicit exploration and openness to new information and activities (Bowlby, 1988; Feeney, 2007; Mikulincer \& Shaver, 2007), which in turn are likely to enhance feelings of personal growth in the long-run.
One noteworthy feature of existing studies is that they have focused on diverse indicators of well-being including affect, life satisfaction, goal attainment, personal growth, feelings of autonomy, and openness to daily life challenges. Extant theorizing and empirical work indicate that these relatively distinct indicators of well-being can be parsimoniously described along two dimensions: hedonia and eudaimonia. Hedonic well-being primarily concerns the experience of pleasure and pain, and is typically operationalized as the extent to which one is satisfied with their life and experiences positive affect (Diener, Suh, Lucas, \& Smith, 1999). In contrast, eudaimonic well-being primarily reflects the extent to which one achieves their potential, and finds meaning and purpose in life. Studies with large and diverse samples of adults (Gallagher, Lopez, \& Preacher, 2009; Keyes, Shmotkin, \& Ryff, 2002) confirm this theoretical distinction. Specifically, different indicators of well-being are hierarchically organized under two positively correlated but empirically distinct latent factors representing hedonic and eudaimonic well-being.

Theoretically, both forms of well-being should be bolstered by perceived partner responsiveness. However, considering fairly recent historic changes in the functions people ascribe to marriage, one would expect perceived partner responsiveness to be a particularly important process for eudaimonic well-being. For instance, the suffocation model of marriage in America (Finkel, Hui, Carswell, \& Larson, 2014) argues that people in the U.S. have become increasingly less dependent on marriage for meeting their basic needs-such as economic well-being, domestic production, safety, and belonging in the community. Instead, marriage is increasingly more important, the model claims, for supporting spouses' autonomy, personal growth, purpose, and self-actualization needs-that is, for helping each other achieve eudaimonic well-being. Yet, actively supporting another individual's eudaimonic well-being is not easy. It requires a deep understanding of the needs of that individual, appreciating that those needs are important, and enacting behaviors supporting those needs-in short, being responsive to that person. Therefore, one hypothesis following from the suffocation model is that perceived partner responsiveness should be a unique predictor of eudaimonic well-being in the United States.

A recent study (Selcuk, Gunaydin, Ong, \& Almeida, 2016) examining the associations between perceived partner responsiveness and both forms of well-being using data from a nationally representative sample of adults in the Midlife Development in the United States (MIDUS) cohort supported this prediction. Perceived partner responsiveness concurrently predicted greater eudaimonic well-being (measured by autonomy, personal growth, purpose in life, self-acceptance, and environmental mastery) and hedonic well-being (measured by life satisfaction, positive affect, and negative affect) at MIDUS I. However, in a 10-year follow up, initial levels of perceived partner responsiveness uniquely predicted increases only in eudaimonic (but not hedonic) well-being from MIDUS I to MIDUS II. These findings provide support for the suffocation model by documenting the unique role of perceived partner responsiveness in predicting eudaimonic well-being in the United States. However, whether relationship processes such as perceived partner responsiveness are equally important for wellbeing in non-Western contexts is an open question. 


\section{Cultural Moderation of the Predictive Role of Perceived Partner Responsiveness in Well-Being}

On one hand, there are theoretical perspectives suggesting that perceived partner responsiveness would be equally relevant for well-being across different countries. For instance, according to attachment theory, responsiveness of attachment figures-parents in early life and romantic partners in adulthood-is an evolutionarily favored process for effective emotion regulation (Beckes \& Coan, 2015), and hence for better personal well-being. One expectation that would follow from this argument is that the predictive role of perceived partner responsiveness in well-being would generalize to different countries.

On the other hand, there is also reason to think that the predictive role of perceived partner responsiveness and well-being would vary across countries. A comparison between the United States and Japan would be relevant here because of the contrasting view of the self in relation to others (self-construals). Individuals in North America are thought to have independent self-construals, where the self is the primary source of psychological experience and relationships are secondary to the self. In contrast, individuals in Japan are thought to have interdependent self-construals, where relational connection is prioritized over the self (Adams, Anderson, \& Adonu, 2004; Markus \& Kitayama, 2010). These two views of the social world are likely to lead to meaningful differences in how people experience close relationships, what they value in relationship partners, and how relationships influence personal well-being.

At first glance, the differences in self-construals and the greater emphasis on relational harmony in the Japanese (vs. U.S.) culture as a predictor of happiness (Uchida \& Kitayama, 2009) may lead one to expect that perceived partner responsiveness would be a stronger predictor of well-being in Japan than in the United States. However, a closer consideration of the foundations of marital and romantic relationships in the two countries suggests that perceived partner responsiveness may in fact more strongly predict wellbeing in the United States than in Japan. The perspective on romantic relationships in independent cultures promotes personal choice and voluntary interaction with potential partners (Adams et al., 2004). This viewpoint may lead individuals to form specific preferences about desirable characteristics (e.g., responsiveness) in potential mates and to believe that success of the relationship depends on these characteristics. In contrast, the role of situational factors (such as the preferences of the kin, (dis)approval of the existing social network, or fate) rather than personal control in relationship formation is more pronounced in interdependent cultures (e.g., Goodwin \& Findlay, 1997). According to this view, individuals' experiences and desires in their relationship are less critical for relationship development and maintenance than situational factors beyond their control. Thus, partner traits are considered secondary to the experience of relationships and possibly to individual well-being. Indeed, individuals from independent cultural backgrounds (e.g., North America, Europe) rate themselves higher on traits reflecting responsiveness (e.g., caring, supportive, affectionate) and desire these traits in a potential partner more so than individuals from interdependent cultural backgrounds (e.g., East Asia, Ghana; Goodwin, 1999; Goodwin et al., 2012).

The belief that partners' behaviors (rather than external factors) are critical determinants of relationship success may lead
American adults to also behave in ways that may elicit responsiveness. American adults self-disclose in their romantic relationships more than their Japanese counterparts (Kito, 2005). Greater self-disclosure, whether it is negative or positive in content, affords more opportunities for responsive behavior (Laurenceau et al., 1998; Otto et al., 2015). Indeed, one study found that daily perceptions of being understood and appreciated (core components of responsiveness) was lower among Asian (vs. Caucasian) participants, possibly because Asian individuals self-disclose less (Oishi, Akimoto, Richards, \& Suh, 2013). To the extent that responsive behaviors successfully attenuate the discloser's negative affect when disclosing a negative issue (e.g., sharing a personal problem) and prolong the discloser's positive affect when disclosing a positive issue (e.g., sharing a personal success), perceived partner responsiveness may more strongly predict personal well-being for American (vs. Japanese) adults.

Finally, cultures where romantic relationships are seen as a product of volitional personal choice are also characterized by higher residential mobility and living away from long-term friends and family (Oishi, 2010; Salter \& Adams, 2012). These living arrangements, combined with lower time spent with other social network members following marriage (Finkel et al., 2014), make the romantic relationship the prototypical and main source of responsive support. In contrast, cultures where romantic relationships are seen as a product of factors external to the self are characterized by low residential mobility and living in close proximity to social network members including family and long-term friends. In such settings, perhaps due to high frequency of contact with the existing social network members, romantic relationships may be less emphasized as the primary source of responsiveness. Reflecting these differences, East Asians are more likely than Americans to cite biological family (over romantic partners) as the primary source of emotional support (Goodwin, 1999) and to report greater preference for helping their family (vs. romantic partner) if they are to choose one of them ( $\mathrm{Wu}, \mathrm{Cross}, \mathrm{Wu}, \mathrm{Cho}$, \& Tey, 2016). Although these findings suggest that perceived partner responsiveness would less strongly predict well-being in Japan than in the United States, this possibility has yet to be empirically tested.

\section{Present Research}

In the current research, we aimed to fill an important gap in the literature by investigating whether the predictive role of perceived partner responsiveness in well-being varies across Japan and the United States. Greater desire and expectations of partner responsiveness, greater self-disclosure, and prioritizing romantic ties as a source of responsiveness are relatively more common in the United States, and thus are all likely to strengthen the role of perceived partner responsiveness in well-being. Accordingly, we hypothesized that perceived partner responsiveness would more strongly predict well-being in the United States than in Japan. Given recent theorizing on the increasing importance of marriage in the United States for meeting eudaimonic needs (Finkel et al., 2014), we expected the cross-country difference to be particularly pronounced for eudaimonic well-being. In line with prior crosscultural work on relationships and well-being (e.g., Kitayama, Karasawa, Curhan, Ryff, \& Markus, 2010), our analyses controlled 
for demographic factors (age, gender, education) as well as extraversion and neuroticism - the major personality predictors of wellbeing (Keyes et al., 2002).

\section{Method}

\section{Sample}

The data were drawn from two large-scale studies, one conducted in the United States and the other in Japan. The U.S. sample was from the second wave of the Midlife Development in the United States (MIDUS II) survey, conducted in 20042006 (Ryff et al., 2007). The Japanese sample was from the Midlife Development in Japan (MIDJA) survey, conducted in 2008-2010. (Ryff, Kitayama, Karasawa, Markus, Kawakami, \& Coe, 2008). The MIDUS II was designed as a 10-year follow-up to the original MIDUS study. The MIDJA was designed as parallel to the MIDUS II to test whether behavioral and psychosocial predictors of mental and physical health in middle and late adulthood differ across the United States and Japan. The MIDJA participants were randomly selected from residents of the Tokyo metropolitan area and the data were collected in the year of 2008 .

MIDUS II consisted of a phone interview followed by a self-administered survey. Four thousand forty-one individuals participated in both the phone interview and the selfadministered survey. The self-administered survey contained a section where participants evaluated their marriage. Participants were asked to complete this section if they were married or cohabiting with a partner, otherwise to skip this section. Nine hundred sixty-two participants skipped this section, leaving 3,079 respondents who identified themselves as married or cohabiting at the time of completing the survey. In the MIDJA sample, 861 (out of 1,027) respondents completed the section evaluating their marriage. Thus, the total analytic sample included 3,940 married or cohabiting adults. In the U.S. sample, mean age was 55 years (range $=33-83$ years), $51 \%$ of respondents were female and $49 \%$ were male, $32 \%$ of respondents graduated from high school or less and $68 \%$ had some college education or more. In the Japanese sample, mean age was 56 years (range $=30-79$ years), $52 \%$ of respondents were female and $48 \%$ were male, $57 \%$ of respondents graduated from high school or less and $43 \%$ had some college education or more. There was no difference between the two samples in terms of gender composition or mean age (both $p s>.103$ ). However, in line with general trends in educational attainment (e.g., United Nations Development Programme, 2014), a greater percentage of U.S. respondents pursued higher education than Japanese respondents, $\chi^{2}(1)=179.215, p<.001$, contingency coefficient $=0.209$.

\section{Procedure and Measures}

MIDUS respondents provided demographic information (age, gender, and education) during a phone interview, and completed the remaining measures (perceived partner responsiveness, hedonic and eudaimonic well-being, and personality traits) as part of a self-administered survey. MIDJA respondents completed all measures as part of a self-administered survey. ${ }^{1}$ MIDJA survey items were created using translation-back translation, with native speakers making adjustments where necessary to ensure analogous meaning. All procedures and measures were reviewed and approved by the University of Wisconsin-Madison Institutional Review Board (Protocol SE-2006-00612 for MIDUS II and 20110458 for MIDJA).

Perceived partner responsiveness. Following prior work (e.g., Selcuk et al., 2016; Selcuk, Stanton, Slatcher, \& Ong, 2017), perceived partner responsiveness was assessed with three items in the MIDUS and MIDJA self-administered questionnaires. The items addressed the core features of responsiveness identified in the extant literature (Reis, 2012) and asked participants to indicate how much they think their partner really cared for, understood, and appreciated them. Respondents answered these questions on a 4-point scale $(1=$ a lot to $4=$ not at all, $\alpha=.836$ in the U.S. sample and $1=$ not at all to $4=$ a lot, $\alpha=.904$ in the Japanese sample). For all continuous variables included in the present study, responses were reversecoded where necessary so that higher scores reflected higher standing on a given variable in both the U.S. and Japanese samples. (For perceived partner responsiveness, $M=3.592$ and $S D=0.564$ for the U.S. sample and $M=2.807$ and $S D=0.754$ for the Japanese sample.)

Well-being. In line with prior studies (Gallagher et al., 2009; Selcuk et al., 2016), well-being was assessed in terms of both its eudaimonic and hedonic components.

Hedonic well-being. Hedonic well-being was conceptualized as the degree to which participants were satisfied with their life and the frequency with which they felt positive and negative affect. Life satisfaction was measured by a single item asking participants to rate their life overall on a scale ranging from 0 (worst) to 10 (best). Positive and negative affect were measured with instruments specifically developed for the MIDUS/MIDJA projects based on items from existing well-validated affect and well-being inventories (e.g., Watson, Clark, \& Tellegen, 1988) Participants rated how much of the time they felt several positive and negative affective states during the previous 30 days on a 5 -point scale $(1=$ all of the time to $5=$ none of the time in the U.S. sample and $1=$ none of the time to $5=$ all of the time in the Japanese sample). The 10-item positive affect scale included feeling "cheerful," "full of life," "in good spirits," "extremely happy," "satisfied," "calm and peaceful," "attentive," "proud," "enthusiastic," and "active." The 11-item negative affect scale included feeling "upset," "so sad nothing could cheer you up," "hopeless," "that everything was an effort," "worthless," "nervous," "jittery," "restless or fidgety," "irritable," "afraid," and "ashamed." We computed a composite hedonic well-being score by reverse-scoring negative affect, standardizing each subscale using pooled standard deviations (i.e., standard deviations were calculated within each country and then averaged; Cohen, 1992) so that affect and life satisfaction measures had the same scaling, and then averaging across the scales $(\alpha=.776, M=0.130, S D=0.770$ for the U.S. sample and $\alpha=.698, M=-0.473, S D=0.850$ for the Japanese sample).

\footnotetext{
${ }^{1}$ All data and materials have been made publicly available and can be accessed at https://www.icpsr.umich.edu/icpsrweb/ICPSR/series/203
} 
Eudaimonic well-being. Respondents completed Ryff's (1989) scales of Psychological Well-Being, a widely used instrument based on a comprehensive operationalization of eudaimonic well-being. The instrument assesses six facets of eudaimonic well-being including autonomy (e.g., "I have confidence in my opinions even if they are contrary to the general consensus"), environmental mastery (e.g., "In general, I feel I am in charge of the situation in which I live"), personal growth (e.g., "For me, life has been a continuous process of learning, changing, and growth"), positive relations with others (e.g., "Most people see me as loving and affectionate"), purpose in life (e.g., "I have a sense of direction and purpose in life"), and self-acceptance (e.g., "When I look at the story of my life, I am pleased with how things have turned out"). Each subscale included seven items rated on a 7-point scale $(1=$ strongly agree to $7=$ strongly disagree in the U.S. sample and $1=$ strongly disagree to $7=$ strongly agree in the Japanese sample). In the present investigation, we excluded the positive relations with others subscale from the analyses since participants are likely to take into account their marriage when responding to the items, which would artificially inflate the predictive role of perceived partner responsiveness in well-being (see Selcuk et al., 2016 for a similar approach). For all the remaining aspects of eudaimonic well-being, subscale scores were constructed by averaging across items. To facilitate comparisons of the slopes across the two forms of well-being, we standardized the eudaimonic well-being dimensions using pooled standard deviations (as we did for hedonic well-being) and then computed a composite eudaimonic well-being score by averaging across the dimensions $(\alpha=.878, M=0.220$, $S D=0.927$ for the U.S. sample and $\alpha=.848, M=-0.792, S D=$ 0.672 for the Japanese sample).

\section{Covariates}

Demographic covariates. Demographic covariates included age, gender $(0=$ male, $1=$ female $)$, and education $(0=$ graduated from high school or less, $1=$ some college education or more).

Personality covariates. Extraversion and neuroticism were measured by the Midlife Development Inventory Personality Scales (Lachman \& Weaver, 1997) developed for the MIDUS project. The measure consists of 25 self-descriptive adjectives that were selected from previous inventories of major personality traits (e.g., John, 1990). Participants indicated how much each adjective described them on a 4-point scale $(1=a$ lot to $4=$ not at all in the U.S. sample and $1=$ not at all to $4=a$ lot in the Japanese sample). Neuroticism scale included four adjectives-moody, calm (reverse-coded), worrying, and nervous $(\alpha=.746, M=2.062, S D=0.622$ for the U.S. sample and $\alpha=.484, M=2.070, S D=0.535$ for the Japanese sample) and extraversion subscale included five adjectives-outgoing, friendly, lively, active, talkative $(\alpha=.756, M=3.103, S D=$ 0.564 for the U.S. sample and $\alpha=.837, M=2.450, S D=$ 0.670 for the Japanese sample).

\section{Missing Data}

In the U.S. sample, missing data were very low: $0.1 \%$ in education, $0.5 \%$ in personality traits, $0.7 \%$ in perceived partner responsiveness, $0.2 \%$ in eudaimonic well-being, and none in the remaining variables. In the Japanese sample, missing data were also very low ( $1.4 \%$ in education, $0.5 \%$ in neuroticism, $0.3 \%$ in extraversion, and none in the remaining variables) except for perceived partner responsiveness where missing data were $10.7 \%$. For all analyses, missing data were imputed via LISREL's multiple imputation module using the expected maximization algorithm.

\section{Measurement Invariance of Variables}

Comparison of regression slopes across groups (in our case perceived partner responsiveness predicting well-being across the U.S. and Japan) require establishing configural and loading invariance (Chen, 2007). Configural invariance reveals that the measures have the same factor structures across groups, and loading invariance (also called metric invariance) reveals that the indicators of the factors have the same loadings across groups. When loading invariance of all indicators is not satisfied (as it is common in many psychology studies; Byrne, Shavelson, \& Muthen, 1989), crossgroup comparisons can still be made if there are only one or a few noninvariant items because those items will not affect the comparisons of slopes to any meaningful degree (Byrne et al., 1989; Cheung \& Rensvold, 2002) — a situation referred to as partial loading invariance. We tested both configural and loading invariance via multigroup confirmatory factor analyses using LISREL. A number of simulation studies examined the best-performing fit indices when testing for measurement invariance (e.g., Chen, 2007; Cheung \& Rensvold, 2002). This work revealed that configural invariance is likely to be rejected when root mean square error of approximation (RMSEA) exceeds 0.05 (Cheung \& Rensvold, 2002) and loading invariance is likely to be rejected when the difference between the comparative fit index (CFI) of the configural model and that of the loading model $(\Delta \mathrm{CFI})$ exceeds 0.01 (Chen, 2007). In line with these results and recommendations in the field of measurement (Wu, Li, \& Zumbo, 2007), we used RMSEA $\leq 0.05$ criterion to evaluate configural invariance and $\Delta \mathrm{CFI} \leq 0.01$ to evaluate loading invariance. If $\Delta \mathrm{CFI}$ exceeded the 0.01 threshold, we relaxed the equality constraint on one item to test for partial loading invariance (see Whisman \& Judd, 2016 for a similar approach). We tested measurement invariance for each construct independently as recommended by Chen (2008).

First, we examined invariance in eudaimonic well-being by treating it as a latent factor and its five dimensions (autonomy, environmental mastery, personal growth, purpose in life, and selfacceptance) as indicators. Given the conceptual and operational overlap, the errors between autonomy and environment mastery, and the errors between purpose in life and personal growth were allowed to correlate. The results supported both configural invariance $(\mathrm{RMSEA}=0.045)$ and loading invariance $\left(\mathrm{CFI}_{\text {Configural }}=\right.$ 0.998 vs. $\left.\mathrm{CFI}_{\text {Loading }}=0.994 ; \Delta \mathrm{CFI}=0.004\right)$. The analyses treating hedonic well-being as the latent factor and life satisfaction, positive affect, and reverse-scored negative affect as manifest indicators also demonstrated configural invariance (RMSEA = 0.000 ) but the test for loading invariance with all indicator loadings constrained to be equal revealed a decrease in CFI that slightly exceeded the recommended 0.01 threshold $\left(\mathrm{CFI}_{\text {Configural }}=1.000 \mathrm{vs}\right.$. $\left.\mathrm{CFI}_{\text {Loading }}=0.985 ; \Delta \mathrm{CFI}=0.015\right)$. When we removed the equality constraint on life satisfaction, partial loading invariance was established $\left(\mathrm{CFI}_{\text {Configural }}=1.000\right.$ vs. $\mathrm{CFI}_{\text {Loading }}=0.997$; $\Delta \mathrm{CFI}=0.003)$. The analyses treating perceived partner responsiveness as the latent factor, and its three items as indicators 
Table 1

Correlations Among Study Variables

\begin{tabular}{|c|c|c|c|c|c|c|c|c|}
\hline Variable & 1 & 2 & 3 & 4 & 5 & 6 & 7 & 8 \\
\hline 1. Eudaimonic well-being & - & $.575^{* * *}$ & $.324^{* * * *}$ & -.060 & -.022 & $.258^{* * * *}$ & $.541^{* * * *}$ & $-.309^{* * * *}$ \\
\hline 2. Hedonic well-being & $.665^{* * * *}$ & - & $.353^{* * * *}$ & .048 & $.069^{*}$ & $.097^{* *}$ & $.401^{* * * *}$ & $-.394^{* * * *}$ \\
\hline 3. Perceived partner responsiveness & $.331^{* * *}$ & $.383^{* * *}$ & - & -.046 & $-.106^{* *}$ & $.093^{* *}$ & $.220^{* * * *}$ & $-.068^{*}$ \\
\hline 4. Age & $.064^{* * * *}$ & $.172^{* * * *}$ & $.134^{* * *}$ & - & -.048 & $-.236^{* * *}$ & $-.077^{*}$ & $-.259^{* * * *}$ \\
\hline 5. Gender ${ }^{\mathrm{a}}$ & $-.061^{* * *}$ & $-.044^{*}$ & $-.145^{* * *}$ & $-.095^{* * * *}$ & - & $-.213^{* * *}$ & .033 & $-.079^{*}$ \\
\hline 6. Education ${ }^{\mathrm{b}}$ & $.172^{* * * *}$ & $.070^{* * * *}$ & .004 & $-.119^{\text {***** }}$ & $-.048^{* *}$ & - & $.094^{* * *}$ & -.012 \\
\hline 7. Extraversion & $.483^{* * * *}$ & $.408^{* * * *}$ & $.175^{* * *}$ & $.053^{* *}$ & $.067^{* * *}$ & -.011 & - & .068 \\
\hline 8. Neuroticism & $-.493^{* * *}$ & $-.537^{* * * *}$ & $-.175^{* * * *}$ & $-.177^{\text {****** }}$ & $.124^{* * * *}$ & $-.098^{* * * *}$ & $-.195^{* * * *}$ & - \\
\hline
\end{tabular}

Note. Estimates below the diagonal reflect associations in the U.S. sample, and those above the diagonal reflect associations in the Japanese sample. All continuous variables were standardized using pooled standard deviations.

${ }^{\mathrm{a}} 0=$ male, $1=$ female. ${ }^{\mathrm{b}} 0=$ high school graduation or less, $1=$ some college education or more.

${ }^{*} p<.05$. *** $p<.01$. **** $p<.001$.

supported configural invariance (RMSEA $=0.000$ ), but loading invariance test with all item loadings constrained to be equal revealed a decrease in CFI that exceeded the recommended threshold $\left(\mathrm{CFI}_{\text {Configural }}=1.000\right.$ vs. $\left.\mathrm{CFI}_{\text {Loading }}=0.951 ; \Delta \mathrm{CFI}=0.049\right)$. When we relaxed the loading equality constraint for the item "How much does your partner really care about you?" partial loading invariance was established $\left(\mathrm{CFI}_{\text {Configural }}=1.000 \mathrm{vs} . \mathrm{CFI}_{\text {Loading }}=\right.$ $0.991 ; \Delta \mathrm{CFI}=0.009)$. With respect to personality covariates, the analyses treating extraversion as the latent factor and the five items as indicators (with the error terms between items "outgoing" and "active," and between "lively" and "active" allowed to correlate as suggested by modification indices) revealed configural invariance $($ RMSEA $=0.044)$, but loading invariance test with all item loadings constrained to be equal revealed a decrease that slightly exceeded the recommended threshold $\left(\mathrm{CFI}_{\text {Configural }}=0.997\right.$ vs. $\left.\mathrm{CFI}_{\text {Loading }}=0.983, \Delta \mathrm{CFI}=0.014\right)$. When we relaxed the equality constraint on the item "active" partial loading invariance was established $\left(\mathrm{CFI}_{\text {Configural }}=0.997\right.$ vs. $\mathrm{CFI}_{\text {Loading }}=0.991, \Delta \mathrm{CFI}=$ 0.006). Finally, analyses with neuroticism as the latent factor and four items as indicators (with the error term between items "worrying" and "nervous" allowed to correlate as suggested by modification indices) provided support for both configural invariance $(\mathrm{RMSEA}=0.037)$ and loading invariance $\left(\mathrm{CFI}_{\text {Configural }}=0.999\right.$ vs. $\left.\mathrm{CFI}_{\text {Loading }}=0.990, \Delta \mathrm{CFI}=0.009\right)$. Overall, these results establish configural and (at least partial) loading invariance of

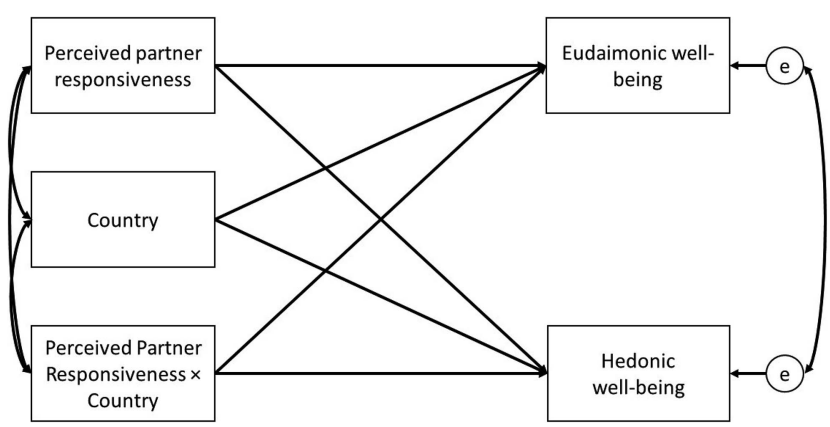

Figure 1. Schematic representation of the path analysis model testing differences in the slope of perceived partner responsiveness predicting well-being across Japan and the United States. measures across the two countries, allowing for comparability of regression slopes.

\section{Results}

Correlations among study variables are provided in Table 1 . We tested the cultural moderation of the role of perceived partner responsiveness in well-being via path analyses using LISREL (see Figure 1 for a schematic representation of the path analytic equations). The path analysis approach allowed us to simultaneously model both forms of well-being as outcomes while controlling for their interrelatedness. In addition to the well-being composites which were already standardized, all continuous predictors were also standardized (using pooled standard deviations) before being entered into the models. We report unstandardized path coefficients reflecting change in well-being as a result of one-unit increase in predictors.

Path analysis treating hedonic and eudaimonic well-being as outcome variables, country $(0=$ United States, $1=$ Japan $)$, perceived partner responsiveness, and their two-way interaction as predictors revealed that country of residence moderated the role of perceived partner responsiveness in both hedonic well-being $(B=-0.098,95 \% \mathrm{CI}=[-0.153,-0.043])$ and eudaimonic well-being $(B=-0.179,95 \% \mathrm{CI}=[-0.241,-0.117]$; see Table $2){ }^{2}$ We probed the nature of the interactions with the online tool described by Preacher, Curran, and Bauer (2006), using the asymptotic covariances of path analytic estimates produced by LISREL. As shown in Figure 2, perceived partner responsiveness positively predicted hedonic and eudaimonic well-being both in the U.S. and Japan. However, perceived partner responsiveness more strongly predicted both types of well-being in the U.S. as compared with Japan $(B=0.362,95 \%$ CI: $[0.331,0.393]$ vs. $B=$ $0.264,95 \%$ CI: $[0.219,0.309]$ for hedonic well-being, $B=0.374$, $95 \%$ CI: $[0.338,0.410]$ vs. $B=0.195,95 \%$ CI: $[0.144,0.246]$ for eudaimonic well-being).

Next, we tested whether the difference in the slopes of perceived partner responsiveness predicting eudaimonic well-being was greater than that predicting hedonic well-being. We repeated the

\footnotetext{
${ }^{2}$ The findings remained the same when we repeated the analysis by removing the error covariance term between eudaimonic and hedonic well-being.
} 
Table 2

Path Analytic Estimates Predicting Well-Being

\begin{tabular}{|c|c|c|c|c|c|c|c|c|}
\hline \multirow[b]{2}{*}{ Predictor } & \multicolumn{4}{|c|}{ Eudaimonic well-being } & \multicolumn{4}{|c|}{ Hedonic well-being } \\
\hline & $B$ & $S E$ & $p$ & $95 \% \mathrm{CI}$ & $B$ & $S E$ & $p$ & $95 \% \mathrm{CI}$ \\
\hline \multicolumn{9}{|c|}{ Model with no covariates ${ }^{\mathrm{a}}$} \\
\hline Perceived partner responsiveness & .374 & .018 & $<.001$ & {$[.339, .409]$} & .362 & .016 & $<.001$ & {$[.331, .393]$} \\
\hline Country ${ }^{\mathrm{b}}$ & -.745 & .040 & $<.001$ & {$[-.823,-.667]$} & -.276 & .035 & $<.001$ & {$[-.345,-.207]$} \\
\hline Perceived Partner Responsiveness $\times$ Country & -.179 & .032 & $<.001$ & {$[-.242,-.116]$} & -.098 & .028 & $<.001$ & {$[-.153,-.043]$} \\
\hline \multicolumn{9}{|c|}{ Model with demographic and personality covariates ${ }^{\mathrm{c}}$} \\
\hline Perceived partner responsiveness & .236 & .015 & $<.001$ & {$[.207, .265]$} & .246 & .014 & $<.001$ & {$[.219, .273]$} \\
\hline Country ${ }^{\mathrm{b}}$ & -.423 & .033 & $<.001$ & {$[-.488,-.358]$} & -.094 & .030 & .002 & {$[-.153,-.035]$} \\
\hline Age & -.035 & .011 & .002 & {$[-.057,-.013]$} & .035 & .010 & $<.001$ & {$[.015, .055]$} \\
\hline Gender $^{\mathrm{d}}$ & -.015 & .021 & .472 & {$[-.056, .026]$} & .069 & .019 & $<.001$ & {$[.032, .106]$} \\
\hline Education $^{\mathrm{e}}$ & .259 & .022 & $<.001$ & {$[.216, .302]$} & .071 & .021 & $<.001$ & {$[.030, .112]$} \\
\hline Extraversion & .360 & .011 & $<.001$ & {$[.338, .382]$} & .237 & .010 & $<.001$ & {$[.217, .257]$} \\
\hline Neuroticism & -.307 & .011 & $<.001$ & {$[-.329,-.285]$} & -.310 & .010 & $<.001$ & {$[-.330,-.290]$} \\
\hline Perceived Partner Responsiveness $\times$ Country & -.148 & .025 & $<.001$ & {$[-.197,-.099]$} & -.048 & .023 & .036 & {$[-.093,-.003]$} \\
\hline
\end{tabular}

Note. All continuous variables were standardized using pooled standard deviations before being entered into the models.

${ }^{\mathrm{a}}$ Error covariance for eudaimonic and hedonic well-being $=.357(S E=.011) .{ }^{\mathrm{b}} 0=$ United States, $1=$ Japan. ${ }^{\mathrm{c}}$ Error covariance for eudaimonic and hedonic well-being $=.151(S E=.006) .{ }^{\mathrm{d}} 0=$ male, $1=$ female. ${ }^{\mathrm{e}} 0=$ high school graduation or less, $1=$ some college education or more.

path analysis by constraining the effect of the interaction term on hedonic and eudaimonic well-being to be equal. This resulted in an 8.804 points increase in model $\chi^{2}(d f=1, p=.003)$, indicating that the difference in the slopes predicting eudaimonic well-being was greater than the difference in the slopes predicting hedonic well-being.

Finally, we tested whether perceived partner responsiveness by country interaction was robust against inclusion of covariates. The two sets of covariates were entered gradually in separate models. We first entered demographic covariates. The difference in the slopes of perceived partner responsiveness predicting well-being across the U.S. and Japan remained the same adjusting for age, gender, and education $(B=-0.088,95 \% \mathrm{CI}=[-0.142,-0.034]$ for hedonic well-being and $B=-0.186,95 \% \mathrm{CI}=[-0.247,-0.125]$ for eudaimonic well-being). Next, we added personality covariates. The difference in the slopes was also robust to inclusion of extraversion and neuroticism $(B=-0.048,95 \% \mathrm{CI}=[-0.093,-0.003]$ for hedonic well-being, and $B=-0.148,95 \% \mathrm{CI}=[-0.197,-0.099]$ for eudaimonic well-being; see Table 2). ${ }^{3}$

\section{Discussion}

Over the last 2 decades, considerable evidence has shown that marital or long-term romantic relationships robustly predict better psychological well-being and physical health (Proulx, Helms, \& Buehler, 2007; Robles, Slatcher, Trombello, \& McGinn, 2014). However, this work has been almost entirely limited to Western contexts, leaving open the question of whether the role of marital processes in well-being would depend on cultural context. This question is increasingly important given the changes in the functions of marriage in North America within the last 50 years, with many individuals now increasingly viewing marriage as a way to satisfy eudaimonic needs, which makes relationship processes such as perceived partner responsiveness more and more central for marital and personal well-being (Finkel et al., 2014). This change leads one to expect that perceived partner responsiveness would be a unique relationship resource enhancing eudaimonic well-being in the long-run, a prediction supported by recent empirical work (Selcuk et al., 2016). But is perceived partner responsiveness equally critical for well-being in other countries? The present study was the first to address this question.

We found that perceived partner responsiveness positively predicted hedonic and eudaimonic well-being both in the U.S. and in Japan. However, the prediction was stronger for U.S. respondents than their Japanese counterparts. The difference in the slopes persisted even after we controlled for demographic factors (age, gender, and education) and personality traits (extraversion and neuroticism) known to affect well-being.

These findings have important theoretical implications given that perceived partner responsiveness is a key construct appearing in almost all major theories of interpersonal relationships (e.g., attachment theory, interdependence theory, social support theory, risk regulation theory; see Reis, 2013 for a review). Of all these theories, probably the one that has been subjected to the most rigorous cross-cultural tests (e.g., Schmitt at al., 2004) is Bowlby's

\footnotetext{
${ }^{3}$ The MIDUS/MIDJA questionnaires also included items assessing perceived network responsiveness (i.e., family and friends). However, configural invariance analysis revealed a very high RMSEA (0.401), indicating that perceived network responsiveness may have different factor structures across the two countries. Thus, we were not able to include this variable in the main analyses. Nevertheless, for informational purposes, we ran a separate model controlling for demographics, personality traits, and perceived network responsiveness. After additionally controlling for perceived network responsiveness, the interaction between country and perceived partner responsiveness still predicted eudaimonic well-being $(B=-0.123,95 \% \mathrm{CI}=[-0.172,-0.074])$. However, for hedonic well-being, the formerly steeper slope in the U.S. got weaker to a similar level as in Japan and the interaction term was no longer significant ( $B=-0.032,95 \% \mathrm{CI}=[-0.077,0.013])$. This pattern of findings is in line with the propositions of the suffocation model such that in the U.S perceived network responsiveness can compensate for perceived partner responsiveness in hedonic, but not eudaimonic, well-being (see also Selcuk et al., 2016). Nevertheless, given lack of measurement invariance for perceived network responsiveness, readers should take these findings with caution until replicated with invariant measures.
} 


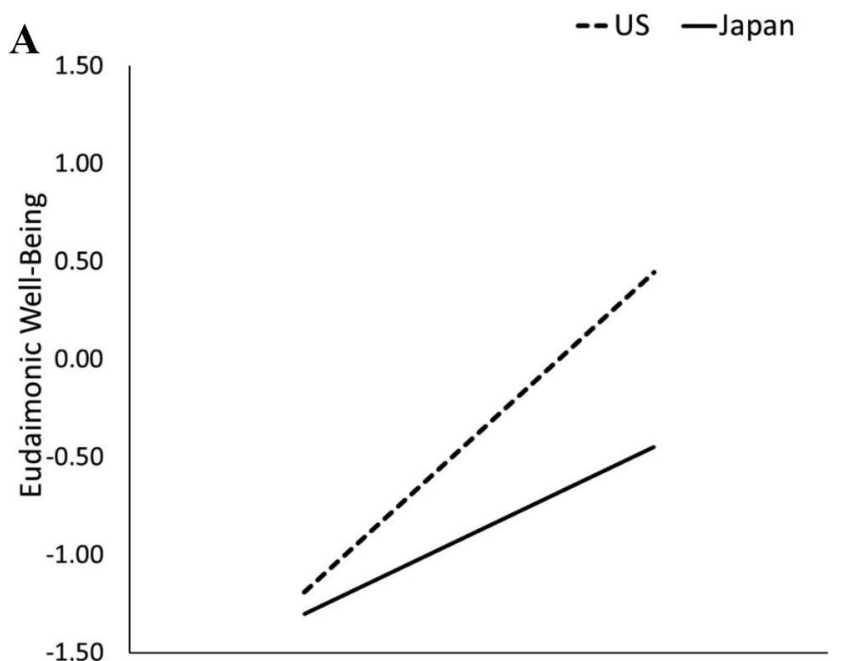

$-1.50$
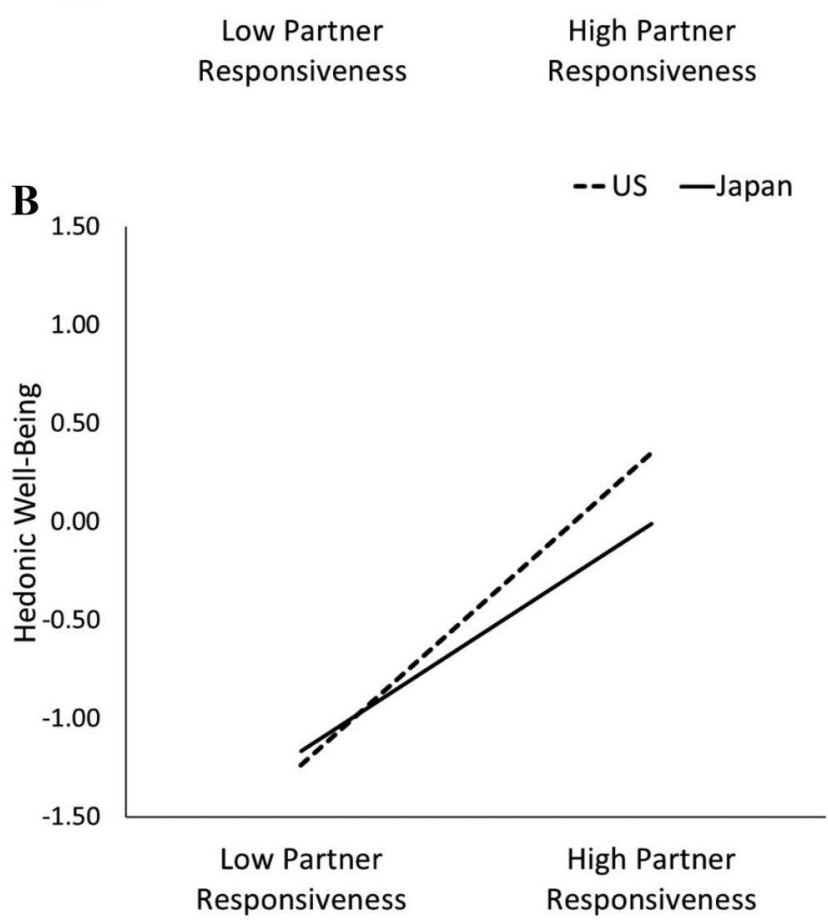

Figure 2. Predicting eudaimonic well-being (Panel A) and hedonic wellbeing (Panel B) from perceived partner responsiveness across the United States and Japan. Both the predictor and outcomes were standardized using pooled standard deviations.

(1988) attachment theory. This theory argues that perceived responsiveness of close others as a base of security to explore the world, pursue personally meaningful goals, work toward achieving the ideal-self, and grow as a person (hallmarks of eudaimonia) is universally essential for healthy human development from the cradle to the grave (Selcuk, Zayas, \& Hazan, 2010). Our finding that perceived partner responsiveness positively predicted both forms of well-being in both the U.S. and Japan supports the idea that perceived partner responsiveness is a fundamental process for personal well-being across diverse cultural contexts.

Nevertheless, consistent with cultural constructions of romantic relationships and marriage in the East versus the West (e.g.,
Adams et al., 2004) as well as recent demographic and sociological shifts in the U.S. (Finkel et al., 2014), we also found that perceived partner responsiveness more strongly predicted well-being for U.S. as compared with Japanese respondents, and this difference was more pronounced for eudaimonic as compared with hedonic wellbeing.

How big is the difference in the slopes of perceived partner responsiveness in predicting well-being across the U.S. and Japan? Given that all variables in the path analytic models were standardized, one can use Cohen's (1992) criteria to interpret the size of the interaction effects. The cross-country difference we observed was small for hedonic well-being and small-to-medium for eudaimonic well-being. This supports the idea that the well-being benefits of perceived partner responsiveness may be universal but we think the differences are nevertheless meaningful as predicted by the differences in cultural constructions of marriage in the two countries as well as changing demographic and sociological factors surrounding marriage in the U.S. (Finkel et al., 2014). Whether the greater predictive role of perceived partner responsiveness in well-being (especially eudaimonia) is unique to the U.S. or the relatively smaller slope is unique to Japan calls for future cross-cultural work testing the role of perceived partner responsiveness in well-being in other parts of the world.

Given that perceived partner responsiveness less strongly predicts well-being for Japanese adults, are there other characteristics of marriage that would be stronger predictors of well-being in Eastern (vs. Western) cultures? Adams et al. (2004) raised the possibility that meeting social obligations (e.g., kin keeping) may be a more important indicator of closeness and intimacy in nonWestern settings, which we speculate, in turn would predict personal well-being perhaps more so than perceived partner responsiveness (see also Imamoglu \& Selcuk, in press). We are aware of no studies that investigated responsiveness to one's needs versus social obligations as unique predictors of well-being. Such studies will not only advance our understanding of the complex pathways by which marriage influences well-being across cultures but also help refine theoretical arguments on how relationships shape normative human development.

Recent work using the MIDUS data has shown that the relevance of perceived partner responsiveness for personal wellbeing also encompasses various indicators of physical health including sleep behavior (Selcuk et al., 2017), diurnal cortisol functioning (Slatcher, Selcuk, \& Ong, 2015), and all-cause mortality (Selcuk \& Ong, 2013). Whether country of residence also moderates the extent to which perceived partner responsiveness predicts physical health is another important question for future research

Before concluding, limitations of the present investigation should be noted. Although the MIDUS sample covered the coterminous United States, the MIDJA sample was drawn only from the Tokyo metropolitan area, limiting the generalizability of the findings to all of Japan. Generalizability of the findings within the U.S. is also limited given that MIDUS sample was primarily White. Future work comparing the role of perceived partner responsiveness in well-being across individuals with different ethnic backgrounds will help increasing the generalizability of the current findings. In addition, the U.S. sample was larger than the Japanese 
sample, which resulted in more statistical power for estimations in the U.S. sample.

These limitations notwithstanding, the study has important strengths. Major theoretical perspectives in mainstream psychology commonly described perceived responsiveness as a fundamental and universal human need (e.g., Bowlby, 1988; Reis, 2013). Evidence supporting this theorizing has largely emanated from Western countries, leaving the universality argument unaddressed. Important work in recent years has started to fill this gap with respect to the role of perceived social network support in well-being (Kitayama et al., 2010; Uchida, Kitayama, Mesquita, Reyes, \& Morling, 2008). The present work contributes to this effort by focusing on a close relationshipmarriage-with particularly potent effects on well-being and comparing for the first time the role of perceived partner responsiveness in personal well-being in middle and late adulthood across the U.S. and Japan. Our findings showed that perceived partner responsiveness positively predicted wellbeing in both countries, but also revealed some meaningful cross-country differences with the role of perceived partner responsiveness being stronger for U.S. as compared with Japanese participants. The large sample size afforded adequate power to test the moderation hypothesis and increased confidence in standard error estimates. Our findings open up interesting avenues for future work on how culture shapes the ways in which individuals satisfy the basic need for relationality and the mechanisms by which relationships affect personal wellbeing, and therefore, contribute to achieving a deeper understanding of interpersonal flourishing across the world.

\section{References}

Adams, G., Anderson, S. L., \& Adonu, J. K. (2004). The cultural grounding of closeness and intimacy. In D. J. Mashek \& A. Aron (Eds.), The handbook of closeness and intimacy (pp. 321-339). Mahwah, NJ: Lawrence Erlbaum.

Beckes, L., \& Coan, J. A. (2015). The distress-relief dynamic in attachment bonding. In V. Zayas \& C. Hazan (Eds.), Bases of adult attachment: Linking brain, mind and behavior (pp. 11-33). New York, NY: Springer.

Bowlby, J. (1988). A secure base: Clinical applications of attachment theory. London, UK: Routledge.

Byrne, B. M., Shavelson, R. J., \& Muthén, B. (1989). Testing for the equivalence of factor covariance and mean structures: The issue of partial measurement invariance. Psychological Bulletin, 105, 456-466. http://dx.doi.org/10.1037/0033-2909.105.3.456

Chen, F. F. (2007). Sensitivity of goodness of fit indexes to lack of measurement invariance. Structural Equation Modeling, 14, 464-504. http://dx.doi.org/10.1080/10705510701301834

Chen, F. F. (2008). What happens if we compare chopsticks with forks? The impact of making inappropriate comparisons in cross-cultural research. Journal of Personality and Social Psychology, 95, 1005-1018. http://dx.doi.org/10.1037/a0013193

Cheung, G. W., \& Rensvold, R. B. (2002). Evaluating goodness-of-fit indexes for testing measurement invariance. Structural Equation Modeling, 9, 233-255. http://dx.doi.org/10.1207/S15328007SEM0902_5

Cohen, J. (1992). A power primer. Psychological Bulletin, 112, 155-159. http://dx.doi.org/10.1037/0033-2909.112.1.155

Collins, N. L., \& Feeney, B. C. (2000). A safe haven: An attachment theory perspective on support seeking and caregiving in intimate relationships. Journal of Personality and Social Psychology, 78, 1053-1073. http://dx .doi.org/10.1037/0022-3514.78.6.1053
Crossley, A., \& Langdridge, D. (2005). Perceived sources of happiness: A network analysis. Journal of Happiness Studies, 6, 107-135. http://dx .doi.org/10.1007/s10902-005-1755-Z

Cutrona, C. E., \& Russell, D. W. (2017). Autonomy promotion, responsiveness, and emotion regulation promote effective social support in times of stress. Current Opinion in Psychology, 13, 126-130. http://dx .doi.org/10.1016/j.copsyc.2016.07.002

Debrot, A., Cook, W. L., Perrez, M., \& Horn, A. B. (2012). Deeds matter: Daily enacted responsiveness and intimacy in couples' daily lives. Journal of Family Psychology, 26, 617-627. http://dx.doi.org/10.1037/ a0028666

Diener, E., Suh, E. M., Lucas, R. E., \& Smith, H. L. (1999). Subjective well-being: Three decades of progress. Psychological Bulletin, 125 276-302. http://dx.doi.org/10.1037/0033-2909.125.2.276

Feeney, B. C. (2004). A secure base: Responsive support of goal strivings and exploration in adult intimate relationships. Journal of Personality and Social Psychology, 87, 631-648. http://dx.doi.org/10.1037/0022 3514.87.5.631

Feeney, B. C. (2007). The dependency paradox in close relationships: Accepting dependence promotes independence. Journal of Personality and Social Psychology, 92, 268-285. http://dx.doi.org/10.1037/00223514.92.2.268

Feeney, B. C., \& Collins, N. L. (2015). A new look at social support: A theoretical perspective on thriving through relationships. Personality and Social Psychology Review, 19, 113-147. http://dx.doi.org/10.1177/ 1088868314544222

Fekete, E. M., Stephens, M. A. P., Mickelson, K. D., \& Druley, J. A. (2007). Couples' support provision during illness: The role of perceived emotional responsiveness. Families, Systems \& Health, 25, 204-217. http://dx.doi.org/10.1037/1091-7527.25.2.204

Finkel, E. J., Hui, C. M., Carswell, K. L., \& Larson, G. M. (2014). The suffocation of marriage: Climbing Mount Maslow without enough oxygen. Psychological Inquiry, 25, 1-41. http://dx.doi.org/10.1080/ 1047840X.2014.863723

Furnham, A., \& Cheng, H. (2000). Lay theories of happiness. Journal of Happiness Studies, 1, 227-246. http://dx.doi.org/10.1023/A:10100 27611587

Gable, S. L., Gosnell, C. L., Maisel, N. C., \& Strachman, A. (2012). Safely testing the alarm: Close others' responses to personal positive events. Journal of Personality and Social Psychology, 103, 963-981. http://dx .doi.org/10.1037/a0029488

Gable, S. L., \& Reis, H. T. (2010). Good news! Capitalizing on positive events in an interpersonal context. In M. P. Zanna (Ed.), Advances in experimental social psychology (pp. 195-257). San Diego, CA: Elsevier Academic Press. http://dx.doi.org/10.1016/S0065-2601(10)42004-3

Gable, S. L., Reis, H. T., Impett, E. A., \& Asher, E. R. (2004). What do you do when things go right? The intrapersonal and interpersonal benefits of sharing positive events. Journal of Personality and Social Psychology, 87, 228-245. http://dx.doi.org/10.1037/0022-3514.87.2.228

Gallagher, M. W., Lopez, S. J., \& Preacher, K. J. (2009). The hierarchical structure of well-being. Journal of Personality, 77, 1025-1050. http:// dx.doi.org/10.1111/j.1467-6494.2009.00573.x

Goodwin, R. (1999). Personal relationships across cultures. London, England: Routledge. http://dx.doi.org/10.4324/9780203434161

Goodwin, R., \& Findlay, C. (1997). "We were just fated together" . . Chinese love and the concept of yuan in England and Hong Kong. Personal Relationships, 4, 85-92. http://dx.doi.org/10.1111/j.1475-6811 1997.tb00132.x

Goodwin, R., Marshall, T., Fülöp, M., Adonu, J., Spiewak, S., Neto, F., \& Hernandez Plaza, S. (2012). Mate value and self-esteem: Evidence from eight cultural groups. PLoS ONE, 7, e36106. http://dx.doi.org/10.1371/ journal.pone.0036106

Imamoglu, E. O., \& Selcuk, E. (in press). Cultural and self-related considerations in relationship well-being: With particular reference to mar- 
riage in Turkey. In C. C. Weisfeld, G. E. Weisfeld, \& L. M. Dillon (Eds.), Psychology of marriage: An evolutionary and cross-cultural view. New York, NY: Lexington.

John, O. P. (1990). The "Big Five" factor taxonomy: Dimensions of personality in the natural language and in questionnaires. In L. A. Pervin (Ed.), Handbook of personality theory and research (pp. 66-100). New York, NY: Guilford Press.

Keyes, C. L. M., Shmotkin, D., \& Ryff, C. D. (2002). Optimizing wellbeing: The empirical encounter of two traditions. Journal of Personality and Social Psychology, 82, 1007-1022. http://dx.doi.org/10.1037/00223514.82.6.1007

Kitayama, S., Karasawa, M., Curhan, K. B., Ryff, C. D., \& Markus, H. R. (2010). Independence and interdependence predict health and wellbeing: Divergent patterns in the United States and Japan. Frontiers in Psychology, 1, 163. http://dx.doi.org/10.3389/fpsyg.2010.00163

Kito, M. (2005). Self-disclosure in romantic relationships and friendships among American and Japanese college students. The Journal of Social Psychology, 145, 127-140. http://dx.doi.org/10.3200/SOCP.145.2.127140

Lachman, M. E., \& Weaver, S. L. (1997). The Midlife Development Inventory (MIDI) Personality Scales: Scale construction and scoring. Waltham, MA: Brandeis University Press.

Laurenceau, J. P., Barrett, L. F., \& Pietromonaco, P. R. (1998). Intimacy as an interpersonal process: The importance of self-disclosure, partner disclosure, and perceived partner responsiveness in interpersonal exchanges. Journal of Personality and Social Psychology, 74, 1238-1251. http://dx.doi.org/10.1037/0022-3514.74.5.1238

Lu, L., \& Gilmour, R. (2004). Culture and conceptions of happiness: Individual oriented and social oriented SWB. Journal of Happiness Studies, 5, 269-291. http://dx.doi.org/10.1007/s10902-004-8789-5

Maisel, N. C., \& Gable, S. L. (2009). The paradox of received social support: The importance of responsiveness. Psychological Science, 20, 928-932. http://dx.doi.org/10.1111/j.1467-9280.2009.02388.x

Markus, H. R., \& Kitayama, S. (2010). Cultures and selves: A cycle of mutual constitution. Perspectives on Psychological Science, 5, 420430. http://dx.doi.org/10.1177/1745691610375557

Mikulincer, M., \& Shaver, P. R. (2007). Attachment in adulthood: Structure, dynamics, and change. New York, NY: Guilford Press.

Oishi, S. (2010). The psychology of residential mobility: Implications for the self, social relationships, and well-being. Perspectives on Psychological Science, 5, 5-21. http://dx.doi.org/10.1177/1745691609356781

Oishi, S., Akimoto, S. A., Richards, J. R. K., \& Suh, E. M. (2013). Feeling understood as a key to cultural differences in life satisfaction. Journal of Research in Personality, 47, 488-491. http://dx.doi.org/10.1016/j.jrp .2013.04.011

Otto, A. K., Laurenceau, J. P., Siegel, S. D., \& Belcher, A. J. (2015). Capitalizing on everyday positive events uniquely predicts daily intimacy and well-being in couples coping with breast cancer. Journal of Family Psychology, 29, 69-79. http://dx.doi.org/10.1037/fam0000042

Pflug, J. (2009). Folk theories of happiness: A cross-cultural comparison of conceptions of happiness in Germany and South Africa. Social Indicators Research, 92, 551-563. http://dx.doi.org/10.1007/s11205-0089306-8

Preacher, K. J., Curran, P. J., \& Bauer, D. J. (2006). Computational tools for probing interaction effects in multiple linear regression, multilevel modeling, and latent curve analysis. Journal of Educational and Behavioral Statistics, 31, 437-448. http://dx.doi.org/10.3102/1076998603 1004437

Proulx, C. M., Helms, H. M., \& Buehler, C. (2007). Marital quality and personal well-being: A meta-analysis. Journal of Marriage and the Family, 69, 576-593. http://dx.doi.org/10.1111/j.1741-3737.2007 .00393.x

Reis, H. T. (2012). Perceived partner responsiveness as an organizing theme for the study of relationships and well-being. In L. Campbell \& T. J. Loving (Eds.), Interdisciplinary research on close relationships: The case for integration (pp. 27-52). Washington, DC: American Psychological Association. http://dx.doi.org/10.1037/13486-002

Reis, H. T. (2013). Relationship well-being: The central role of perceived partner responsiveness. In C. Hazan \& M. I. Campa (Eds.), Human bonding: The science of affectional ties (pp. 283-307). New York, NY: Guilford Press.

Reis, H. T., \& Shaver, P. R. (1988). Intimacy as an interpersonal process. In S. Duck (Ed.), Handbook of research in personal relationships (pp. 367-389). London, UK: Wiley.

Robles, T. F., Slatcher, R. B., Trombello, J. M., \& McGinn, M. M. (2014). Marital quality and health: A meta-analytic review. Psychological Bulletin, 140, 140-187. http://dx.doi.org/10.1037/a0031859

Ryff, C. D. (1989). Happiness is everything, or is it? Journal of Personality and Social Psychology, 57, 1069-1081. http://dx.doi.org/10.1037/00223514.57.6.1069

Ryff, C. D., Almeida, D. M., Ayanian, J. S., Carr, D. S., Cleary, P. D., Coe, C., ... Williams, D. (2007). Midlife Development in the United States (MIDUS II), 2004-2006. National archive of computerized data on aging. Retrieved from http://www.icpsr.umich.edu/icpsrweb/NACDA/ studies $/ 4652$

Ryff, C. D., Kitayama, S., Karasawa, M., Markus, H., Kawakami, N., \& Coe, C. (2008). Survey of Midlife Development in Japan (MIDJA), April-September 2008 (ICPSR30822-v2). National archive of computerized data on aging. Retrieved from http://www.icpsr.umich.edu/ icpsrweb/NACDA/studies/30822

Salter, P. S., \& Adams, G. (2012). Mother or wife? An African dilemma tale and the psychological dynamics of sociocultural change. Social Psychology, 43, 232-242. http://dx.doi.org/10.1027/1864-9335/a000124

Schmitt, D. P., Alcalay, L., Allensworth, M., Allik, J., Ault, L., Austers, I., . . Braeckman, J. (2004). Patterns and universals of adult romantic attachment across 62 cultural regions: Are models of self and of other pancultural constructs? Journal of Cross-Cultural Psychology, 35, 367402.

Selcuk, E., Gunaydin, G., Ong, A. D., \& Almeida, D. M. (2016). Does partner responsiveness predict hedonic and eudaimonic well-being? A 10-year longitudinal study. Journal of Marriage and the Family, 78, 311-325. http://dx.doi.org/10.1111/jomf.12272

Selcuk, E., Karagobek, A. B., \& Gunaydin, G. (in press). Responsiveness as a key predictor of happiness: Mechanisms and unanswered questions. In M. Demir \& N. Sumer (Eds.), Close relationships and happiness. New York, NY: Springer.

Selcuk, E., \& Ong, A. D. (2013). Perceived partner responsiveness moderates the association between received emotional support and all-cause mortality. Health Psychology, 32, 231-235. http://dx.doi.org/10.1037/ a0028276

Selcuk, E., Stanton, S. C. E., Slatcher, R. B., \& Ong, A. D. (2017) Perceived partner responsiveness predicts better sleep quality through lower anxiety. Social Psychological \& Personality Science, 8, 83-92. http://dx.doi.org/10.1177/1948550616662128

Selcuk, E., Zayas, V., \& Hazan, C. (2010). Beyond satisfaction: The role of attachment in marital functioning. Journal of Family Theory \& Review, 2, 258-279. http://dx.doi.org/10.1111/j.1756-2589.2010.00061.x

Slatcher, R. B., \& Selcuk, E. (2017). A social psychological perspective on the Links between close relationships and health. Current Directions in Psychological Science, 26, 16-21. http://dx.doi.org/10.1177/096372 1416667444

Slatcher, R. B., Selcuk, E., \& Ong, A. D. (2015). Perceived partner responsiveness predicts diurnal cortisol profiles 10 years later. Psychological Science, 26, 972-982. http://dx.doi.org/10.1177/095679761 5575022 
Uchida, Y., \& Kitayama, S. (2009). Happiness and unhappiness in east and west: Themes and variations. Emotion, 9, 441-456. http://dx.doi.org/10 $.1037 / \mathrm{a} 0015634$

Uchida, Y., Kitayama, S., Mesquita, B., Reyes, J. A. S., \& Morling, B. (2008). Is perceived emotional support beneficial? Well-being and health in independent and interdependent cultures. Personality and Social Psychology Bulletin, 34, 741-754. http://dx.doi.org/10.1177/01461 67208315157

United Nations Development Programme. (2014). Mean years of schooling (of adults). Retrieved from https://data.humdata.org/dataset/mean years_of_schooling_of_adults

Watson, D., Clark, L. A., \& Tellegen, A. (1988). Development and validation of brief measures of positive and negative affect: The PANAS scales. Journal of Personality and Social Psychology, 54, 1063-1070. http://dx.doi.org/10.1037/0022-3514.54.6.1063
Whisman, M. A., \& Judd, C. M. (2016). A cross-national analysis of measurement invariance of the Satisfaction With Life Scale. Psychological Assessment, 28, 239-244. http://dx.doi.org/10.1037/pas0000181

Wu, A. D., Li, Z., \& Zumbo, B. D. (2007). Decoding the meaning of factorial invariance and updating the practice of multi-group confirmatory factor analysis: A demonstration with TIMSS data. Practical Assessment, Research \& Evaluation, 12, 1-26.

Wu, T. F., Cross, S. E., Wu, C. W., Cho, W., \& Tey, S. H. (2016). Choosing your mother or your spouse: Close relationship dilemmas in Taiwan and the United States. Journal of Cross-Cultural Psychology, 47, 558-580. http://dx.doi.org/10.1177/0022022115625837

Received February 2, 2017

Revision received September 27, 2017

Accepted October 5, 2017 